\title{
CONCENTRACIONES DE DESARROLLO RURAL - CDR: UN CASO DE TRANSFERENCIA *
}

\author{
Álvaro Toledo \\ Antonio José Niño \\ Carlos Buriticá G. \\ David Díaz \\ Stella Vecino \\ Lucila Bueno
}

Con el ánimo de plantear algunas inquietudes necesarias para abordar el problema de la tecnología y la transferencia tecnológica en la educación colombiana, presentamos a los asistentes al Seminario estas notas, producto de una reflexión inicial.

Somos concientes de la urgencia de que en el país se abra un amplio debate, en torno a este problema de gran actualidad, ya que es necesario clarificar el proceso de modernización del sistema educativo.

Dicha modernización no debe ser entendida como la importación indiferenciada de modelos que acreditan eficacia en otros contextos o que simplemente presentan una validez formal. Ella debe basarse en la investigación de la realidad nacional, con el objeto de definir la problemática educativa y establecer prioridades para formular soluciones adecuadas.

\section{Introducción}

El reconocimiento oficial sobre las diferencias educativas en las áreas rurales y urbanas así, como la aplicación de planes en materia educativa, se evidencian en el país durante los últimos 15 años.

Como parte de los planes para obviar las dificultades de cumplir el precepto jurídico de educación igual para todos los colombianos, se comenzaron a implementar los "núcleos escolares" en 1961, con miras a lograr una mayor expansión de la escolaridad en el campo y una mayor participación de la comunidad afectada, en el proceso educativo.

A partir de ese momento ha sido constante el reconocer oficialmente las diferencias entre campo y ciudad no sólo en materia educativa, sino en todos los aspectos que se consideran necesarios para lograr un desarrollo económico-social igualitario.

Desde los núcleos escolares y el llamado Plan de Emergencia (Decreto 150 de 1967) que programa diversas modalidades para racionalizar recursos educativos, pasando por la Escuela Unitaria, hasta las escuelas de Funcionamiento Intensivo (educación flexible o desescolarizada), la acción gubernamental ha estado orientada a buscar alternativas de solución, estimulados por demanda creciente de educación, para lograr la extensión de la escolaridad en áreas rurales y urbanas.

Dentro del "Plan de Desarrollo Económico y Social 1971-1974", los planteamientos sobre política educativa explicitan la necesidad de vincular la escolaridad con los requerimientos de capacitación de la fuerza de trabajo.

\footnotetext{
* Esta ponencia fue presentada en el primer Seminario Nacional sobre "Política del país en Transferencia de Tecnología en Educación", en Bogotá, marzo de 1978. 
Tal política, estuvo orientada por los lineamientos generales esbozados por la Misión Naciones Unidas - OIT., que como estrategia para el pleno empleo en el país establecía: "...intensificar enormemente la formación en actividades agrícolas y en artesanía rural, una vez que se hayan tomado decisiones concretas, será importante determinar con precisión estas necesidades. En gran medida, la educación y la formación tendrán que ser distintas de los métodos y programas de estudios más o menos convencionales" ${ }^{110}$.

Los puntos más relevantes de sus planteamientos estratégicos pueden resumirse así:

1. Se deben señalar varios aspectos relativos a la formación de los propios trabajadores agrícolas. En primer lugar, la formación de los jóvenes trabajadores se iniciaría realmente con el programa de enseñanza primaria y secundaria esbozado en el capítulo 15, especialmente si se asegura que, incluso en el sector rural, los niños completen por lo menos un ciclo de cuatro años de estudio, con lo cual en mayor proporción seguirán cursos de enseñanza secundaria con mayor contenido de orientación profesional.

2. Mientras se alcanza ese objetivo, se deberían establecer varios centros de formación profesional con el propósito de dar a los jóvenes una formación práctica y teórica intensiva-apoyada en los principios básicos y en los métodos de trabajo de la técnica moderna destinada a prepararlos para su futura labor como trabajadores agrícolas o como artesanos rurales.

3. El establecimiento rápido de una red de centros como los propuestos serviría por lo menos para detener el desempleo crónico, tan desmoralizante, que fácilmente podría conducir a cierta criminalidad juvenil. La formación profesional, combinada con una nueva orientación de enseñanza primaria, también formaría parte de la política rural y ayudaría a contener la migración de los jóvenes hacía las zonas urbanas ${ }^{111}$.

Tal concepción educativa que hace énfasis en el sector rural e implica un cambio social integrado, fue muy bien expresada por el entonces Ministro de Educación: “...no creo que haya cambio social sin la transformación de la educación, pero tampoco creo que todo el cambio se haga solo con la educación; (...) en la sociedad contemporánea es imposible pensar que un hombre va a poder estar integrado a las realidades de la sociedad en que se halla, si el sistema educativo no le procura una oportunidad de mayor ambición, de mayores perspectivas, y si además, de ofrecerle conocimientos mucho más complejos, no procura también atender a las necesidades de su formación para el empleo, y a la consideración de los aspectos vocacionales de esa formación “112.

\section{Concentraciones de desarrollo rural}

Bajo esta concepción y como intento de ofrecer integralmente los niveles de educación primaria y media en el campo, surgen las Concentraciones de Desarrollo Rural.

Los componentes de la problemática a la cual C.D.R., pretende dar solución son: educación, salud, nutrición, desarrollo agropecuario, recreación y organización de la comunidad $^{113}$.

\footnotetext{
${ }^{110}$ O.I.T., Hacia el Pleno Empleo, un programa para Colombia, preparado por una Misión Internacional, organizada por la O.I.T., publicación del Banco Popular, Bogotá, 1970, p. 259

${ }^{111}$ Ibídem.

${ }^{112}$ MEN: La Educación ante el Congreso, Bogotá, 1972.

${ }^{113}$ Las características del Programa de Concentraciones de Desarrollo Rural ha sido tomada de los diferentes documentos 
El Programa se define como: "El mecanismo operativo que resulta de dos procesos centrales, la integración de los servicios y la participación creciente de la población por ellos servida, para alcanzar mayores niveles de bienestar económico, social y cultural. La concentración concilia a nivel regional las necesidades sentidas de la población con las necesidades objetivas del desarrollo nacional" ${ }^{114}$.

De esto se desprende como objetivos generales:

1. Promover el desarrollo del sector rural.

2. Promover la coordinación e integración de los servicios prestados al sector rural.

3. Lograr el mejoramiento o el cambio de los mecanismos de participación social ${ }^{115}$.

Como objetivos específicos del sector educativo en el programa se plantean:

1. Extender la educación rural ofreciendo un mínimo de cinco grados de escolaridad en toda la zona de influencia y los nueve grados por lo menos en cada una de las Concentraciones en su sede central.

2. Mejorar el sistema escolar tradicional a partir de un sistema nuclear en zonas geográficas con condiciones sociales y económicas similares.

3. integrar los programas escolares de educación básica, dentro de un programa de desarrollo de la comunidad.

4. Extender la cobertura para la prestación de servicios de salud al área rural.

5. Desarrollar programas nutricionales.

6. Capacitación y asistencia técnica apropiada al campesino.

7. Mejorar las condiciones de tenencia de la tierra y aumentar la productividad agrícola como un medio para elevar los niveles de vida de la población campesina.

8. Dar participación a la comunidad y promover su organización.

Otros objetivos no menos importantes son:

-Ofrecer mejores condiciones de vida y capacitación permanente a los maestros.

-Ofrecer a los alumnos y a sus familiares incentivos para asistir y permanecer en la escuela.

-Reducir la amplitud en la escala de edades de los estudiantes de nivel primario.

\footnotetext{
elaborados por el Ministerio de Educación Nacional y del Departamento Nacional de Planeación.

${ }^{114}$ DNP-Programa CDR documento URH-DBOS-027, julio de 1972, p. 7

${ }^{115}$ Estructura operativa y normas legales para e funcionamiento del servicio educativo en la CDR-MEN, febrero 1974 , p. 7.
} 
Estos planteamientos esbozan las dos áreas fundamentales del Proyecto CDR: Sede Central de Servicios y Estructura Educativa.

\section{A. Sede Central de Servicios}

"La noción de concentración no se refiere a una unidad estática sobre la cual converjan los servicios que a nivel local prestan o prestarían las instituciones comprometidas. Se trata fundamentalmente de la cooperación entre los agentes institucionales locales para llevar a cabo acciones integradas con la participación activa y consciente de los miembros de la comunidad".

"El programa tiene como premisa fundamental la integración de los sectores Educación, Agricultura, Salud y Desarrollo de la Comunidad, de tal modo que puedan lograrse economías externas y de administración que eleven el rendimiento social y económico de los gastos públicos. En el país hay suficientes instituciones, para atender estos servicios, pero su eficacia depende de la coordinación y concentración de sus acciones".

"La integración no es un fin en sí mismo ni una determinación automática a la cual deberán someterse todas las instituciones que presten servicios en el área rural, sino más bien un proceso consciente y planeado, dirigido a crear en el medio rural las condiciones básicas para su desarrollo"116.

El concepto de sede central de servicios tiene su origen en las recomendaciones de la Comisión de la OIT que elaboró el documento "Hacia el Pleno Empleo" en el año de 1970.

El concepto de concentración de desarrollo rural como estrategia global de desarrollo ubicado en un área geográfica específica, que pretende irradiar los componentes de modernización a las áreas circundantes, responde a la teoría de los "polos de desarrollo" elaborada por CEPAL para América Latina en la década de 1950.

\section{B. Servicios educativos}

El servicio educativo dentro del Programa Concentraciones de Desarrollo Rural persigue básicamente:

a) Implantar el plan de Educación Básica a nueve (9) años, como aspiración mínima a las metas del sector.

b) Mejorar el sistema escolar tradicional sustituyéndolo por una nueva estrategia: el sistema nuclear.

El servicio educativo en las Concentraciones de Desarrollo Rural, significa la extensión vertical del sistema IN EM, (Instituto Nacional de Educación Media Diversificada) haciendo extensivo al país rural, la posibilidad de recibir una educación planificada según las características regionales.

Nucleación. Es la forma adoptada para establecer y administrar el nuevo tipo de educación que responde a los nuevos contenidos curriculares. Esta consiste en la integración del servicio educativo en una zona geográfica con características socioeconómicas similares. Además, al superarse la anterior escuela aislada, el sistema

\footnotetext{
${ }^{116}$ DNP: Documento Síntesis del Programa de concentraciones de Desarrollo Rural. (URHDBO5-037), Bogotá, Junio 14 de 1973. pp. $5-6$. 
nuclear captará todo el potencial educativo de las comunidades fijándoles la correspondiente responsabilidad. El resultado será un mejor y más amplio servicio educacional, basado en las condiciones regionales, y atendiendo a las necesidades de la familia campesina.

Desde 1945 en el Perú y Bolivia se estableció un programa educativo rural denominado Núcleos Escolares cuya estructura formal inspira en gran parte lo que hoy conocemos como estrategia educativa del programa de Concentraciones de Desarrollo Rural.

El Núcleo consta de una sede central que desarrolla actividades escolares y extraescolares conjuntamente con las sedes seccionales a las cuales subordina.

La experiencia del Perú y Bolivia, dirigida a las comunidades indígenas, se difundió rápidamente como respuesta a problemas sociales en otros países de América Latina, entre ellos Colombia que establece este sistema en 1961 y lo aplica a las zonas afectadas por la violencia política.

Aquí se puede extraer una primera diferencia en cuanto a que el sistema nuclear en Perú y Bolivia buscaba solucionar problemas distintos a los planteados para Colombia. En el Perú aparentemente se trataba de dar asistencia social a zonas marginadas rurales a través de la educación como posibilidad de integración nacional más que de desarrollo económico de las zonas en cuestión. Mientras en lo escolar se trataban objetivos claros de ampliación y extensión de la escolaridad y la promoción del desarrollo cultural, en lo extraescolar no se comprometió la participación activa de los sectores no educativos, únicos capaces de ejecutar en la práctica programas de desarrollo adecuados, concomitantes con los propósitos educativos de promoción social, económica y cultural.

En nuestro medio donde las necesidades de desarrollo económico de las zonas afectadas exigían programas educativos relacionados estrechamente con programas de producción y ocupación, el sistema nuclear se introdujo sin una complementación que refleja el propósito de satisfacer necesidades sentidas de las zonas, que no eran propiamente las educativas. El aparato educativo por mucho que pretenda proyectarse a la comunidad no se basta a si mismo. Muchas veces organiza a las comunidades las cuales acceden pasivamente a cumplir con formalismo, sin ninguna movilización por iniciativa consciente.

Una caracterización hecha en 1971 sobre la falta de complementación de los programas de núcleos escolares expone que... "Las coordinaciones en la mayoría de los casos vienen ejerciéndose en base a las amistades o iniciativa del personal de los núcleos o como resultado de algunos seminarios. A esto se debe en gran parte el fenómeno que se registra con frecuencia de que las ayudas, podría decirse, son por lo general de una naturaleza ocasional"117. La expresión "ayudas... de una naturaleza ocasional" es sintomática del débil carácter del papel de los sectores productivos y de la escasa planificación y compromiso intersectorial.

En lo educativo es evidente la similitud entre el sistema de núcleos escolares y el proyecto de concentraciones. Ambos pretenden extender la escolaridad e impulsar programas de desarrollo de la comunidad. Sin embargo, en el proyecto de concentraciones se destaca la necesidad de impulsar la diversificación de la educación y

\footnotetext{
${ }^{117}$ Consideraciones generales sobre las Concentraciones de Desarrollo Rural por Clara Colbert y Norman Knudson, ICOPLE, Bogotá, 1971, Mimeógrafo. 
su orientación vocacional, así como la coordinación con otros programas de desarrollo rural.

La forma como se dará el servicio educativo dentro del sistema nuclear obedece a los siguientes niveles de coordinación y operación:

Sede Central. Es la unidad responsable de la dirección general de todo el servicio educativo (educación básica regular y educación no escolar) que se presta en el área: además es el epicentro de la coordinación e integración de servicios.

En esta sede central se ofrecerá educación del $6^{\circ}$ al $9^{\circ}$ grados, con programas orientados a la formación ocupacional. El $60 \%$ del currículo es de carácter general y constituye la base de conocimientos comunes para todo el país y un $40 \%$ del área vocacional representa la parte flexible del currículo, que se refiere a la exploración vocacional y su consiguiente orientación, permitiendo su adaptación a las exigencias particulares de cada región.

Esta se establecerá de acuerdo con los siguientes criterios:

- Utilización de áreas en donde funcionen establecimientos educativos de enseñanza agrícola, como núcleos escolares, escuelas vocacionales agrícolas, concentraciones agropecuarias y escuelas hogar.

- En las áreas donde no exista ninguno de los programas mencionados se deben tener en cuenta:

-Que sea un área rural.

-Vías de acceso (carreteables) en buen estado y facilidad de transporte.

-Servicios (agua y luz)

Características del terreno: 1) laborable; 2) construible; 3) extensión: construcciones e instalaciones deportivas (4 has.); 4) granja, de cuerdo a la unidad de explotación definida en la región (mínimo 20 has.).

Es deseable que la granja esté integrada a las construcciones, en caso contrario la distancia será 'hasta de $5 \mathrm{kms}$.; 5) terreno no inundable; topografía plana; inclinación pendiente aproximada, madereras, corriente de agua, accidentes naturales o artificiales.

Que sea un centro de convergencia y de atracción respecto de los núcleos de población (caserío, veredas, etc.).

Factibilidad de consecución de terreno, donado por particulares y/o comunidades, municipio o departamento, al MEN (Ministerio de Educación Nacional) ${ }^{118}$.

${ }^{118}$ DNP: Documento Síntesis... op. cit., pp. 7- 8. 
Por su parte la escuela vocacional prevista es un intento de transplantar al área rural el sistema INEM, que a su vez halla su modelo original en la educación diversificada norteamericana (Escuelas Comprensivas).

Escuela Satélite. Constituye el segundo nivel operativo y depende de la Sede Central directamente. Ofrece educación elemental dividida en grados de $1^{\circ}$ a $5^{\circ}$. Coordina la capacitación para los docentes de las escuelas vinculadas que dependan de ella. En algunos casos ofrecerá facilidades para continuidad de los estudios a los escolares de las escuelas vinculadas que no ofrezcan el ciclo primario completo.

Teniendo en cuenta los criterios fijados para la sede central y en forma simultánea, se escogerán aquellas escuelas ubicadas en veredas, inspecciones o corregimientos que reúnan las siguientes características:

-Que exista una concentración significativa de población escolarizable, que permita la implantación de la escuela graduada o que ya esté funcionando como tal, de propiedad del municipio, donde las ampliaciones y reparaciones no impliquen demasiado costo.

-Que su ubicación permita a los escolares de las pequeñas escuelas vecinas donde se imparta enseñanza hasta el $2^{\circ}$ o $3 e r$ grados de educación primaria, llegar a ella para continuar estudios hasta el quinto grado.

-Que la ubicación de estas escuelas respecto de la sede central permita una fácil comunicación con la misma.

-Estas escuelas deben tener un área entre dos y cinco hectáreas, destinadas una para construcciones e instalaciones deportivas y cuatro para la granja. Además de lo anotado anteriormente se debe tener en cuenta, que los terrenos queden distantes de: basureros, cementerios, caños de aguas negras, hospitales, mataderos, postes de alta tensión, oleoductos, ríos o quebradas con grandes variantes de caudal, vías de tránsito pesado y constante (camiones, buses, tren).

Escuela Vinculada. Constituye el tercer nivel de operatividad. Depende de la Escuela Satélite y representa las células básicas del servicio educativo.

Atiende el nivel de educación elemental, recurriendo nuevamente en muchos casos al sistema de escuela unitaria por razones económicas. La escuela vinculada es aquella ubicada generalmente en zonas rurales con escasa densidad de población, atendida por un solo maestro, con un promedio hasta de cuarenta niños de distintos sexos y edades, agrupados por niveles del $1^{\circ}$ al $5^{\circ}$ grado. ${ }^{119}$

Tanto la escuela unitaria como la escuela graduada son sistemas educativos implantados en el país en diferentes épocas: la escuela unitaria surge de las recomendaciones de la UNESCO en la década del 60 y la escuela graduada en la década del 30 como transplante de la Escuela francesa vigente en la época.

\footnotetext{
${ }^{119}$ DNP: Documento Síntesis... op. cit. pp. 9-10.
} 


\section{Tecnología y transferencia tecnológica}

En torno a la problemática de la tecnología educativa se han planteado diversos enfoques que algunos autores han resumido en dos grandes líneas.

a) La tecnología educativa entendida como ayudas de enseñanza.

b) La tecnología educativa como aplicación de la teoría y análisis de sistemas a la educación.

Un planteamiento que intenta una conceptualización global del fenómeno es el de Clayton quien se refiere a la tecnología como una metodología para hallar soluciones a situaciones problemáticas. Según él, el proceso tecnológico incluye lo siguiente:

a) Definición clara del objetivo.

b) Identificación del problema.

c) Examen del ambiente y de los recursos propios del ambiente.

d) Selección del recurso más apropiado.

e) Experimentación y evaluación de la solución.

En su concepto existe una tendencia a confundir la tecnología con sus productos. Plantea que solo cuando se entienda que la tecnología es una capacidad para lograr los propios objetivos con los propios recursos, podrá lograrse la transferencia de tecnología.

\section{A. El concepto de tecnología}

En nuestro concepto, la tecnología es una instancia mediadora entre la concepción teórica de un problema y su solución.

La concepción teórica del problema, implica por una parte el conocimiento científico del área correspondiente y por otra, la concepción acerca de la sociedad, que determina las metas ideales de la misma y por tanto, los objetivos que debe lograr cualquier solución que se plantee.

En otros términos, esa concepción teórica incluye tanto las metas a lograr como la caracterización de la situación problemática, a la luz de las condiciones específicas del medio social en el que se presenta.

A partir de esta definición, la tecnología aparece como una solución propuesta que debe producir efectos congruentes con las metas planteadas al nivel de la concepción teórica.

Dicha solución es una estructura implementable a nivel empírico. Es decir, articula todos los pasos a dar en su aplicación. 


\section{B. El instrumental de la tecnología}

A nivel de la implementación de dicha estructura se hace necesario todo un complejo de instrumentos técnicos sin los cuales es imposible "poner en marcha" la solución. Este instrumental lo podemos clasificar así:

a) De análisis y seguimiento. Son los instrumentos establecidos para ejercer un control sobre la congruencia de la implementación y su correspondencia en la solución planteada, y sobre los efectos que la práctica va produciendo sobre la realidad a transformar.

En este momento y de acuerdo con el desarrollo actual de la ciencia, el instrumento más utilizado a este nivel es de "Análisis de sistemas".

b) Instrumentos indispensables. Son aquellos sin los cuales es imposible implementar la solución propuesta. Se encuentran entre otros, organización administrativa y financiera, instrumental básico de comunicación y de acción sobre el objeto, etc.

c) Instrumental accesorio. Son aquellos elementos que si bien ayudan a implementar la solución propuesta, no son indispensables.

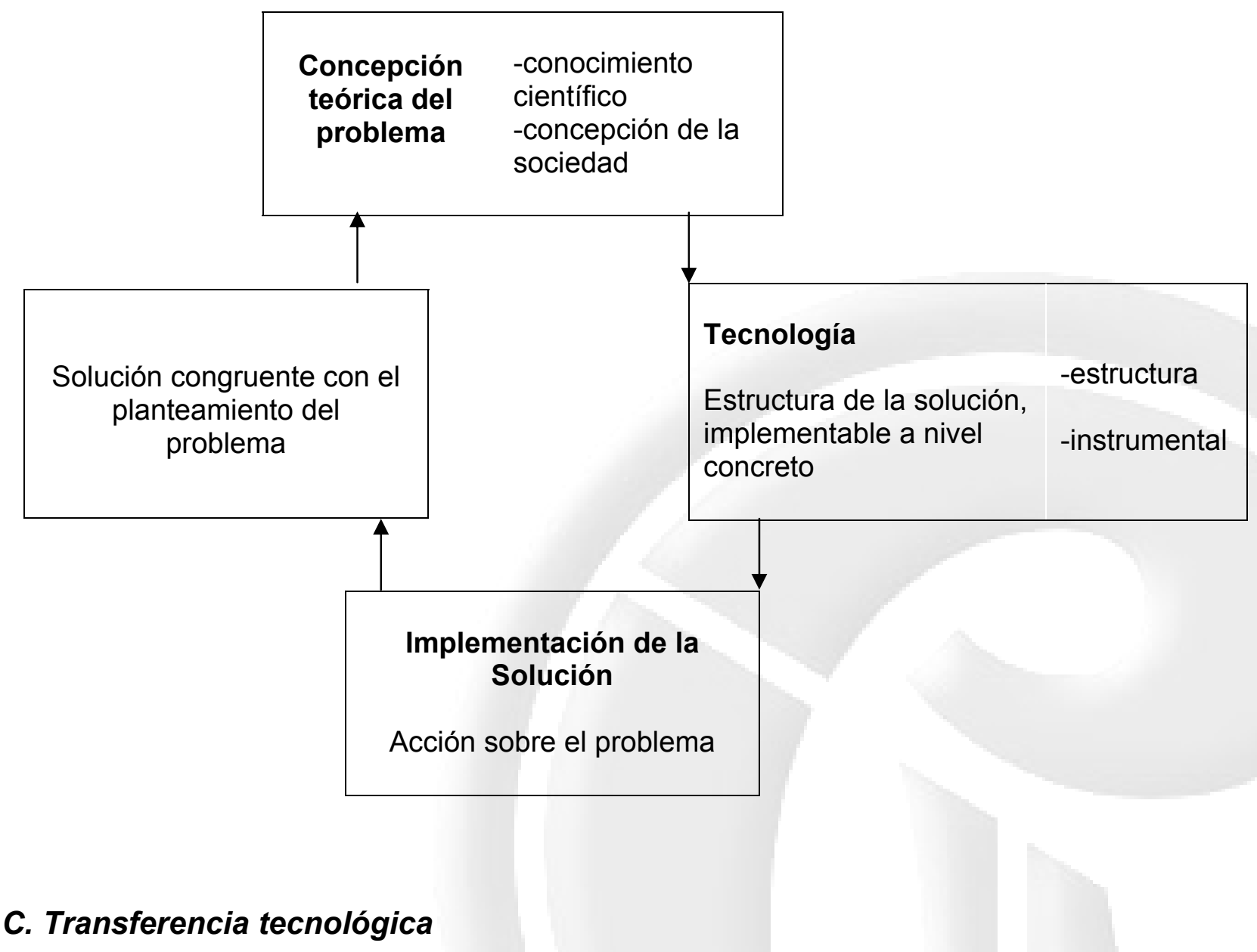

La caracterización del problema revela el vínculo orgánico que existe entre aquél y el medio socioeconómico y cultural en que se da. La solución que se logre adquiere su 
sentido real en el marco de la concepción del problema y el medio socioeconómico y cultural que lo determina.

De acuerdo con este planteamiento, si la solución se aplica en un medio donde la concepción de la sociedad y/o las características del medio socio-económico son diferentes, el resultado no es congruente con las condiciones del problema al que se le pretende aplicar, no siendo por lo tanto solución adecuada al mismo.

Esto no quiere decir, que una solución coherente en un determinado medio no sea aplicable en otro, pero para que esto se de, se requiere de una previa adecuación al mismo. Este proceso de adecuación de una tecnología a un medio diferente a aquél en el cual se originó, es lo que entendemos por TRANSFERENCIA. La aplicación de la solución sin ese proceso, es solo un trasplante cuyos resultados pueden no solamente no dar solución al problema sino que pueden incluso deformar las condiciones del medio al que se aplica.

\section{Concentraciones de desarrollo rural y transferencia tecnológica}

En puntos anteriores de este documento, se estableció cómo las CDR están conformadas por una serie de tecnologías importadas al país en diferentes épocas y situaciones. Hasta qué punto fueron estudiadas y evaluadas para ser integradas en dicho proyecto? Su formulación obedeció a un proceso de transferencia tecnológica? Tuvo en cuenta las condiciones socioeconómicas del país?

El presente trabajo no pretende evaluar el funcionamiento ni los resultados del proyecto de CDR. En consecuencia nos limitamos a examinar algunos aspectos de las CDR a la luz de los planteamientos esbozados acerca de la tecnología y la transferencia tecnológica.

1. En la definición del programa CDR antes citada, se decía que las concentraciones deberían conciliar "a nivel regional las necesidades sentidas de la población con las necesidades objetivas del desarrollo nacional". Este requisito supondría una cuidadosa investigación previa sobre las condiciones socioeconómicas y culturales de las áreas asiento del programa y de su articulación a la dinámica global del país. Sin embargo, parece que esto no llegó a hacerse y más bien la selección de las áreas estuvieron sometidas a criterios de urgencias políticas e institucionales, que impidieron una adecuada identificación y jerarquización de los diferentes aspectos del problema a resolver.

Un aspecto fundamental que resalta del examen de las CDR es el que se refiere a su aplicación en un medio como el rural, sin que aparezca clara su caracterización, su especificidad y la viabilidad de alcanzar las metas propuestas. Un indicador que corrobora los planteamientos anteriores, lo constituye la afirmación acerca de que: "los criterios para selección de área de CDR no tomaron en cuenta prioritariamente la existencia de un potencial de desarrollo agrícola y de acciones en otros campos..."120.

2. En cuanto al instrumental tecnológico para implementar concentraciones de Desarrollo Rural, proyecto elaborado bajo el auspicio de la Cooperación Técnico Internacional dentro del Programa de las Naciones Unidas para el Desarrollo PNUD-

\footnotetext{
${ }^{120}$ Prada. Abner Manuel: Borrador del Informe Final... op. cit., Pág. V.
} 
UNESCO, un aspecto fundamental debió ser la organización administrativa a nivel nacional.

Sin embargo... "La estructura institucional del proyecto Col 72/033 a nivel nacional no fue debidamente precisada...". Esta falta de precisión contribuyó a mantener en situación de permanente inestabilidad al Equipo Técnico encargado de orientar la ejecución del proyecto" $^{\prime 21}$.

Por otra parte, el planteamiento de las CDR como epicentro del desarrollo rural, suponía la implementación de una estructura de planeamiento nacional, que permitiera coordinar e integrar las acciones y recursos de los diferentes sectores estatales, con el objeto de inducir planes integrales a nivel local y del área de influencia de las concentraciones.

La coordinación interinstitucional como fue planteada, no ha operado en la práctica. "Ha sido notoria la ausencia de decisiones capaces de sostener e impulsar la coordinación Inter.-sectorial del proyecto a nivel nacional".

Al no funcionar integralmente la coordinación interinstitucional queda sin piso uno de los componentes básicos del Programa, independientemente de que a partir de las CDR como instancia educativa se puedan realizar algunas acciones hacia la comunidad y de coordinación local.

3. El modelo educativo propuesto es, por su parte, el de la educación básica, de nueve años. Esta se implementa sobre el modelo tradicional: 5 años de educación primaria, sea por medio de la escuela unitaria o graduada, y 4 años de educación diversificada siguiendo el sistema INEM.

No se ha implementado la integración curricular de tal ciclo educativo. Por el contrario, a nivel de los 5 años de educación primaria se sigue conservando el tradicional currículo que se aplica también en áreas urbanas. A nivel de educación media se agregan 4 años de educación vocacional.

Este caso permite observar la incorporación en el modelo, de elementos extraídos de diferentes experiencias sin que las mismas respondan a un sistema integrado coherentemente con la especificidad del medio rural.

Los puntos hasta aquí esbozados sugieren una serie de carencias en el diseño y la implementación del modelo de tecnología educativa implicado en el programa CDR.

Sin embargo la ausencia, en este momento, de una investigación que de cuenta de las condiciones de elaboración del modelo, de sus diferentes modos de adaptación y de sus aspectos operativos, no permiten adelantar un análisis acabado sobre la problemática de la tecnología incorporada al Programa.

${ }^{121}$ Prada. Abner Manuel: Borrador del Informe Final... op. cit., Pág. V. 